Supplementary material to:

\title{
FINDING A CONSENSUS ON CREDIBLE FEATURES AMONG SEVERAL PALEOCLIMATE RECONSTRUCTIONS
}

\author{
By Panu Erästö* ${ }^{*}$, Lasse Holmströ ${ }^{\dagger}$, Atte Korhola $^{\ddagger}$ And Jan \\ WECKSTRÖM ${ }^{\ddagger}$, \\ National Institute for Health and Welfare*, University of Oulu ${ }^{\dagger}$ and \\ University of Helsink $i^{\ddagger}$
}

1. Reconstruction errors. In section 2 of the main paper the model for a reconstructed temperature anomaly $\mathbf{y}_{k}$ is

$$
\mathbf{y}_{k}=\boldsymbol{\mu}_{k}+\varepsilon_{k},
$$

where $\boldsymbol{\mu}_{k}$ is the true temperature and $\varepsilon_{k}$ is the reconstruction error. We report here some exploratory analyses of the properties of the errors $\varepsilon_{k}$ as estimated by the residuals $\hat{\boldsymbol{\varepsilon}}_{k}=\mathbf{y}_{k}-\hat{\boldsymbol{\mu}}_{k}$, where $\hat{\boldsymbol{\mu}}_{k}$ is a smooth of $\mathbf{y}_{k}$. The smooths were computed using local linear regression with a Gaussian kernel where an optimal bandwidth selection method suggested in Ruppert, Sheather and Wand (1995) was employed.

The reconstructed temperature anomalies, their smooths and the associated residuals are depicted in Figure S.1. The bandwidths selected for reconstructions based on Lake Toskal diatoms and pollen were fairly large making the smooths almost linear. The sample standard deviations of the residuals are shown in Table S.1. They are somewhat smaller than the upper bounds $\bar{\sigma}_{k}$ in Table 2 of the main paper but still mostly larger than the alternative "small-error" value 0.2 used in the scale space analysis. A clear exception is the diatom based reconstruction for Lake Tsuolbmajavri for which the residual sample standard deviation is 0.13. Table S.1 indicates that the magnitudes of the reconstruction errors vary quite a bit for the six reconstructions and, on the basis of Figure S.1, at least the errors for the pollen based reconstructions may exhibit heteroskedasticity.

Q-Q plots of the residuals comparing the empirical quantiles of the residuals against standard normal distribution quantiles are displayed in Figure S.2. It appears that the distributions of the residuals could be considered at least roughly normal although a couple of values in the right tails 


\begin{tabular}{lc}
\hline Proxy record & Std of residual \\
\hline Lake Toskal chironomids & 0.19 \\
Lake Toskal diatoms & 0.21 \\
Lake Toskal pollen & 0.54 \\
Lake Tsuolbmajavri chironomids & 0.3 \\
Lake Tsuolbmajavri diatoms & 0.13 \\
Lake Tsuolbmajavri pollen & 0.4 \\
\hline
\end{tabular}

TABLE S.1

Sample standard deviations of the smoothing residuals for the 6 proxy records considered.

of the distributions for Lake Tsuolbmajavri chironomids and pollen appear suspiciously large. Histograms of the residuals are displayed in the diagonal plots of Figure S.4.

To explore error autocorrelation within each reconstruction we examined scatterplots of pairs of residual values. Thus, if the reconstruction errors in (S.1) are $\varepsilon_{k}=\left[\varepsilon_{k 1}, \ldots, \varepsilon_{k j_{k}}\right]^{T}$, we plot the pairs $\left(\hat{\varepsilon}_{k i}, \hat{\varepsilon}_{k, i+1}\right), i=1, \ldots, j_{k}-1$. This is referred to as "lag 1" scatter plot and similar plots were considered for lags 2 and 3. Some evidence for autocorrelation was found for Lake Toskal pollen and Lake Tsuolbma diatoms, both for lag 1 and 2 (cf. Figure S.3).

An attempt was also made to explore dependencies between different reconstructions. This was done by considering scatterplots of residual pairs $\left(\hat{\varepsilon}_{k i}, \hat{\varepsilon}_{l j}\right)$, where $k$ and $l$ denote different reconstructions and $i$ and $j$ correspond to identical dates in the their corresponding chronologies. Even after binning, the chronologies of some reconstructions overlap only partially and in these cases a rather small number of pairs could be plotted using this approach. For example, the overlap between the Lake Toskal Diatom chronology and the Lake Tsuolbma chronology is only 10 dates. The rationale for considering identical dates in the (binned) chronologies is that possible correlations between reconstructions would likely manifest themselves most strongly for such pairs of residuals. The results are shown in Figure S.4. None of the scatterplots were found to exhibit significant correlation, the smallest correlation coefficient $p$-value being 0.29 between Lake Toskal residuals for chironomids and pollen.

These preliminary analyses of the data suggest that the models considered in the main paper are quite reasonable. In particular, a multivariate normal error model for each reconstruction seems to be quite well justified. On the other hand, the more general model that allows prior correlation also between reconstructions may be unnecessarily complex.

2. Additional credibility maps. The feature maps of the main paper were based on the credibility level $\alpha=0.8$. Figures S.5 and S.6 show maps 
based on the credibility level $\alpha=0.95$. As expected, fewer features are flagged as credible. Still, the main features exhibited by the lower credibility maps in Figures 8-10 of the main paper all still clearly visible.

Figures S.7 and S.8 show consensus analyses obtained with the model (6) of the main paper that allows dependencies both within the proxy records and between them. The result for large reconstruction errors (Figure S.7) looks quite similar to the corresponding map in the main paper (Figure 8). The result for small reconstruction errors (Figure S.8) has fewer features than the corresponding map for the simpler model (Figure 9 in the main paper). The exploratory analyses reported above suggested that our data may not require the more complex model and it might be that the more flexible error model interprets some of the actual features as correlated noise. Another explanation are potential convergence problems in the simulations. With the more complex model, computational issues quickly begin to limit the length of feasible MCMC runs. As in the main paper, Figures S.7 and S. 8 here are based on samples of size 4000 where the first 2000 were used for burn-in. Instead of the 10 hours needed in the case of the simpler model, the more complex model required 4 days to generate a sample of this size.

\section{References.}

Fox, J. and Weisberg, S. (2011). An R Companion to Applied Regression, Second ed. Sage, Thousand Oaks CA.

Ruppert, D., Sheather, S. J. and Wand, M. P. (1995). An Effective Bandwidth Selector for Local Least Squares Regression. Journal of the American Statistical Association 90 1257-1270. 

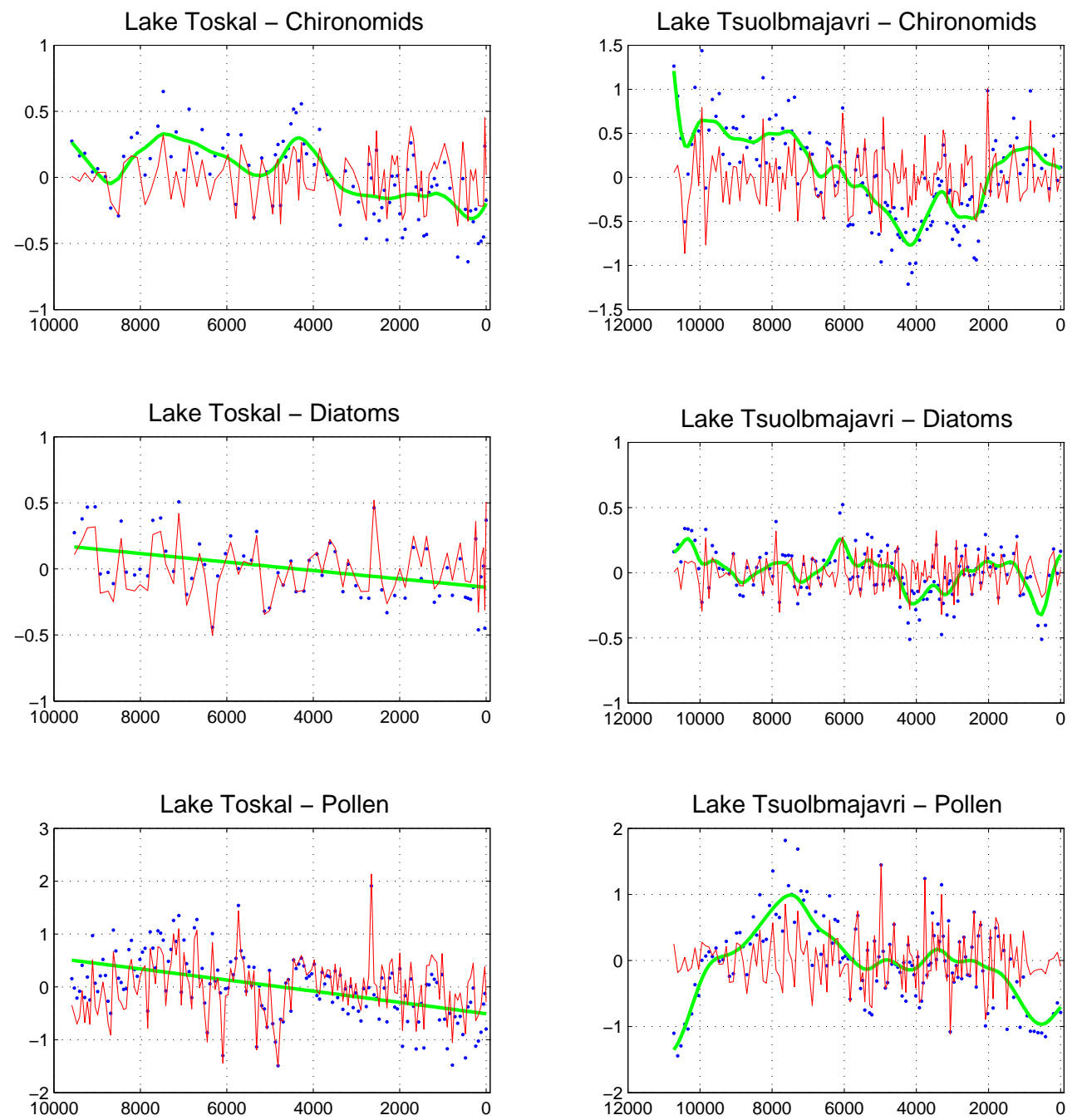

FIGURE S.1. Reconstructed temperature anomalies (blue dots), their smooths (green) and the smoothing residuals (red) computed as their difference. Smooths are local linear regressions with a Gaussian kernel where the bandwidths were computed using a method from Ruppert, Sheather and Wand (1995). 
Lake Toskal - Chironomids

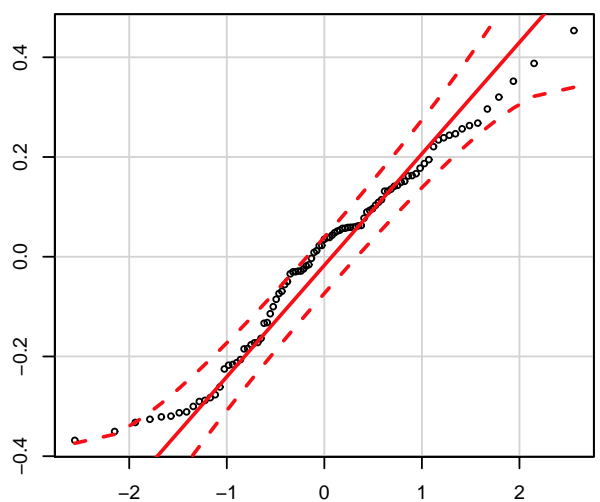

Lake Toskal - Diatoms

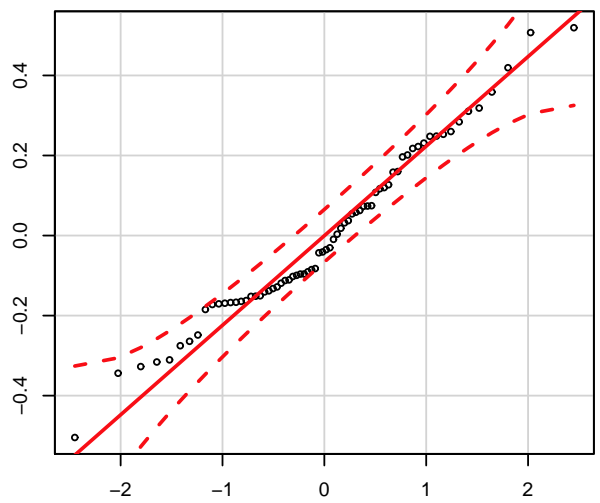

Lake Toskal - Pollen

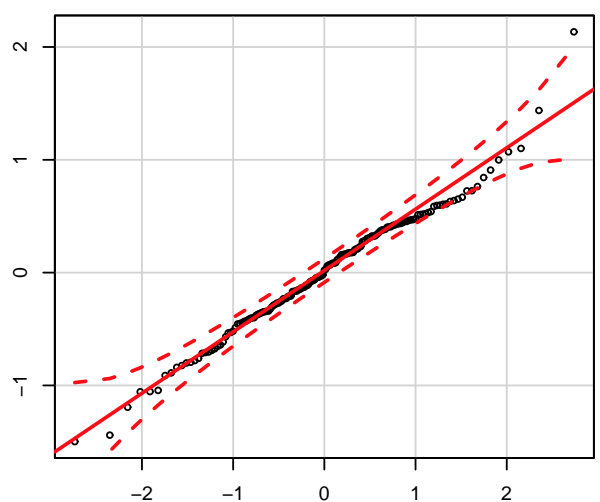

Lake Tsuolbmajavri - Chironomids

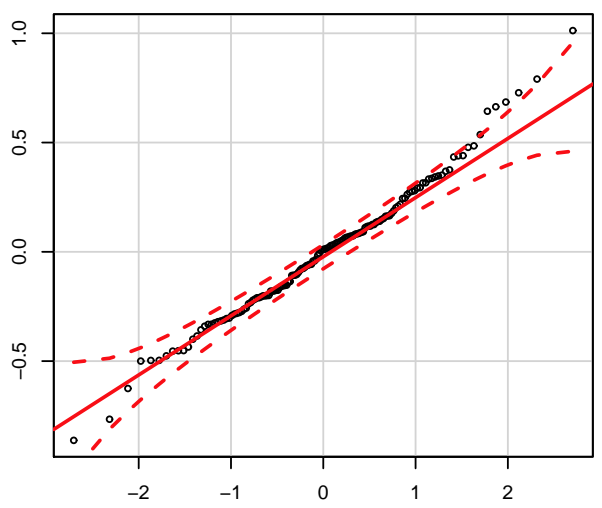

Lake Tsuolbmajavri - Diatoms

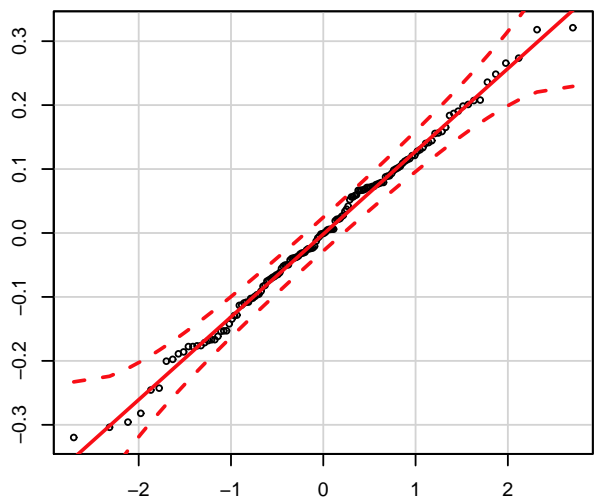

Lake Tsuolbmajavri - Pollen

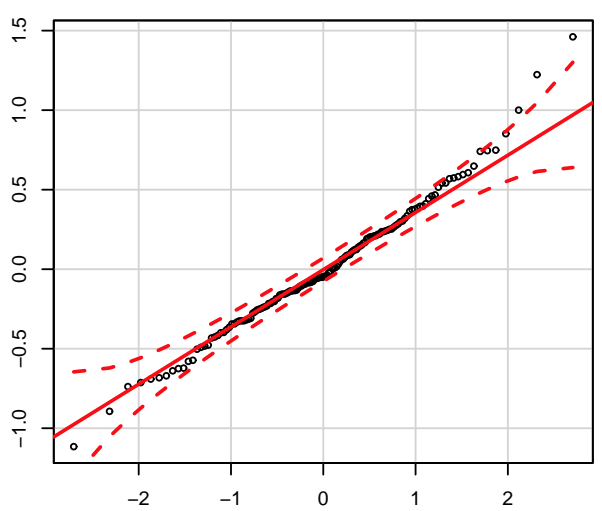

FIGURE S.2. Q-Q plots that compare the empirical quantiles of the reconstruction errors estimated as smoothing residuals (vertical axes) against standard normal distribution quantiles (horizontal axes). The line passes through the first and the third quartiles and the dotted lines show the pointwise $95 \%$ confidence envelope. The plots were produced using the function qqPlot in the car R-package Fox and Weisberg (2011). 

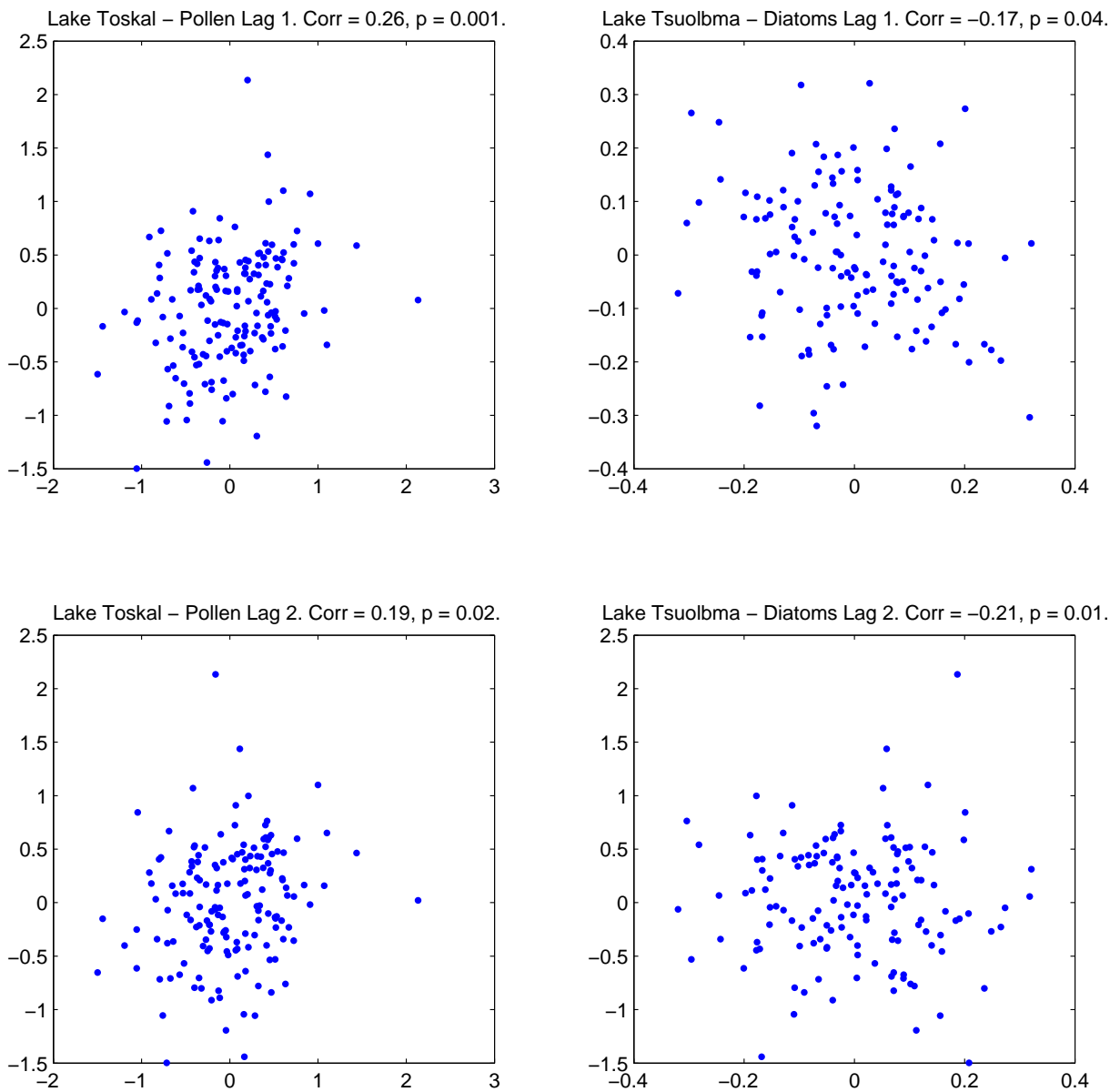

FigURE S.3. Lag 1 and lag 2 scatter plots of reconstruction errors estimated as smoothing residuals for Lake Toskal pollen and Lake Tsuolbma diatom reconstructions. Also given are the estimated correlation coefficients and their p-values. 

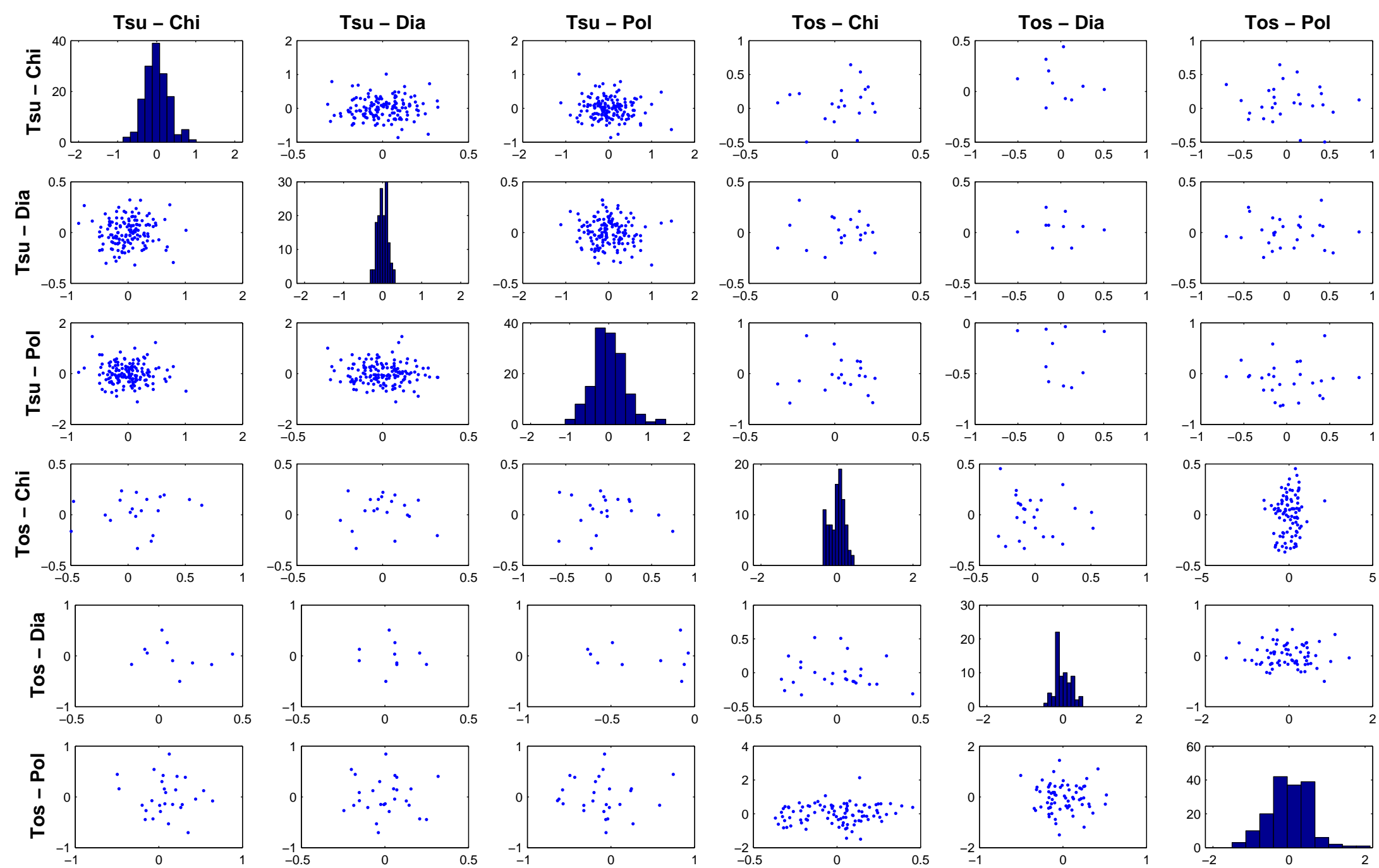

FiguRE S.4. Scatterplots and histograms of the reconstruction errors estimated as smoothing residuals. The dots mark pairs of residuals corresponding to the same dates in the two reconstructions considered. When the chronologies of two reconstructions differ, only errors corresponding to the dates common to the two chronologies are used in the scatter plots. Here "Tsu" and "Tos" stand for Lake Tsuolbmajavri and Lake Toskal, correspondingly, and "Chi", "Dia" and "Pol" stand for Chironomids, Diatoms, and Pollen, respectively. 

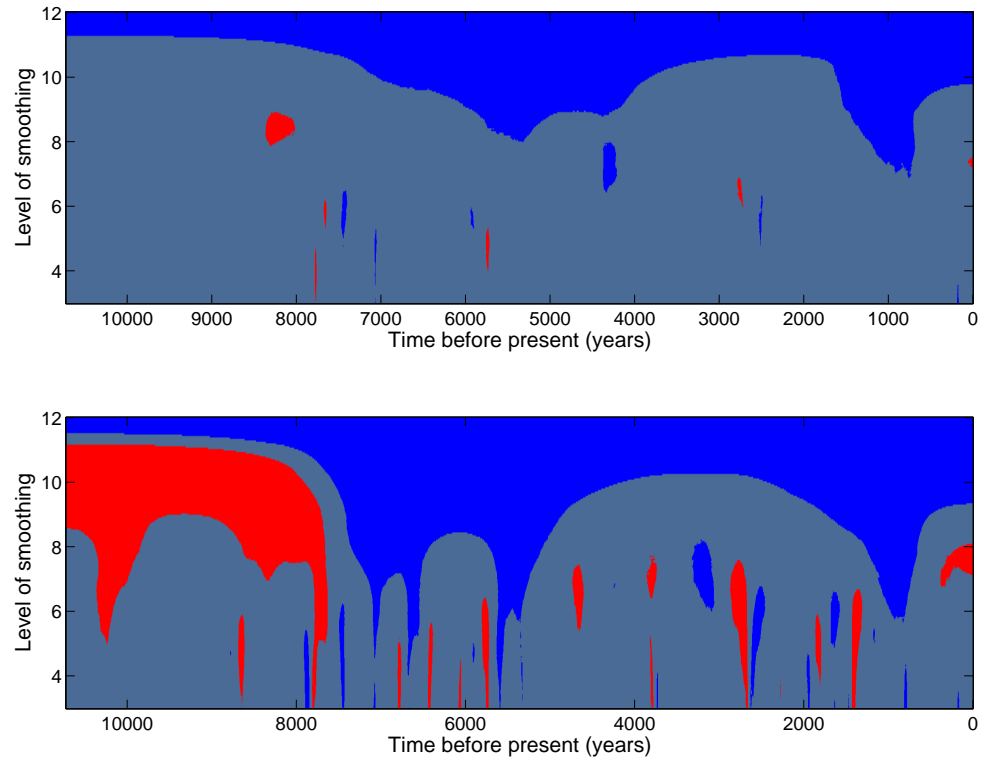

FiguRE S.5. Credibility maps for Holocene temperature consensus with posterior probability level $\alpha=0.95$ when either large (upper panel) or small (lower panel) reconstruction errors are assumed. Blue and red indicate credible cooling and warming, respectively. For more information see the text in the main paper. 

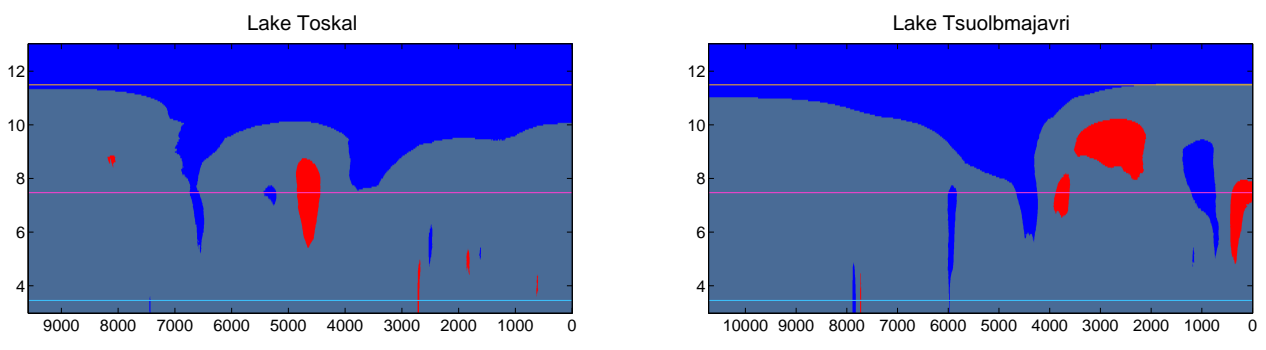

Pollen
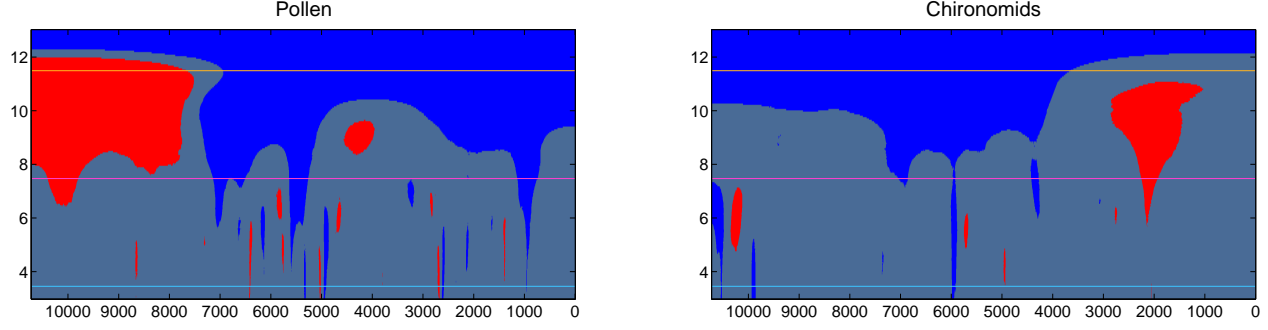

Diatoms

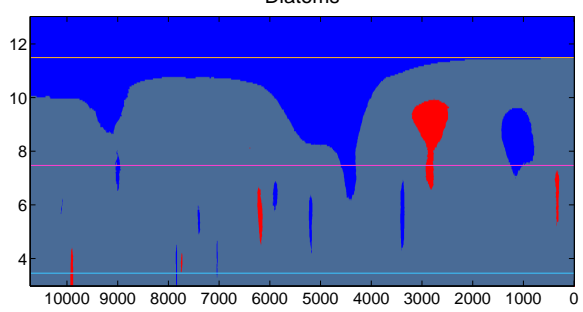

FIGURE S.6. Consensus based on subgroups of the six temperature reconstructions considered. Large reconstruction errors are assumed and the posterior probability level $\alpha=0.95$. In the top row the two lakes are analyzed separately. The other three maps show the consensus according to each proxy. For more information see the text in the main paper. 

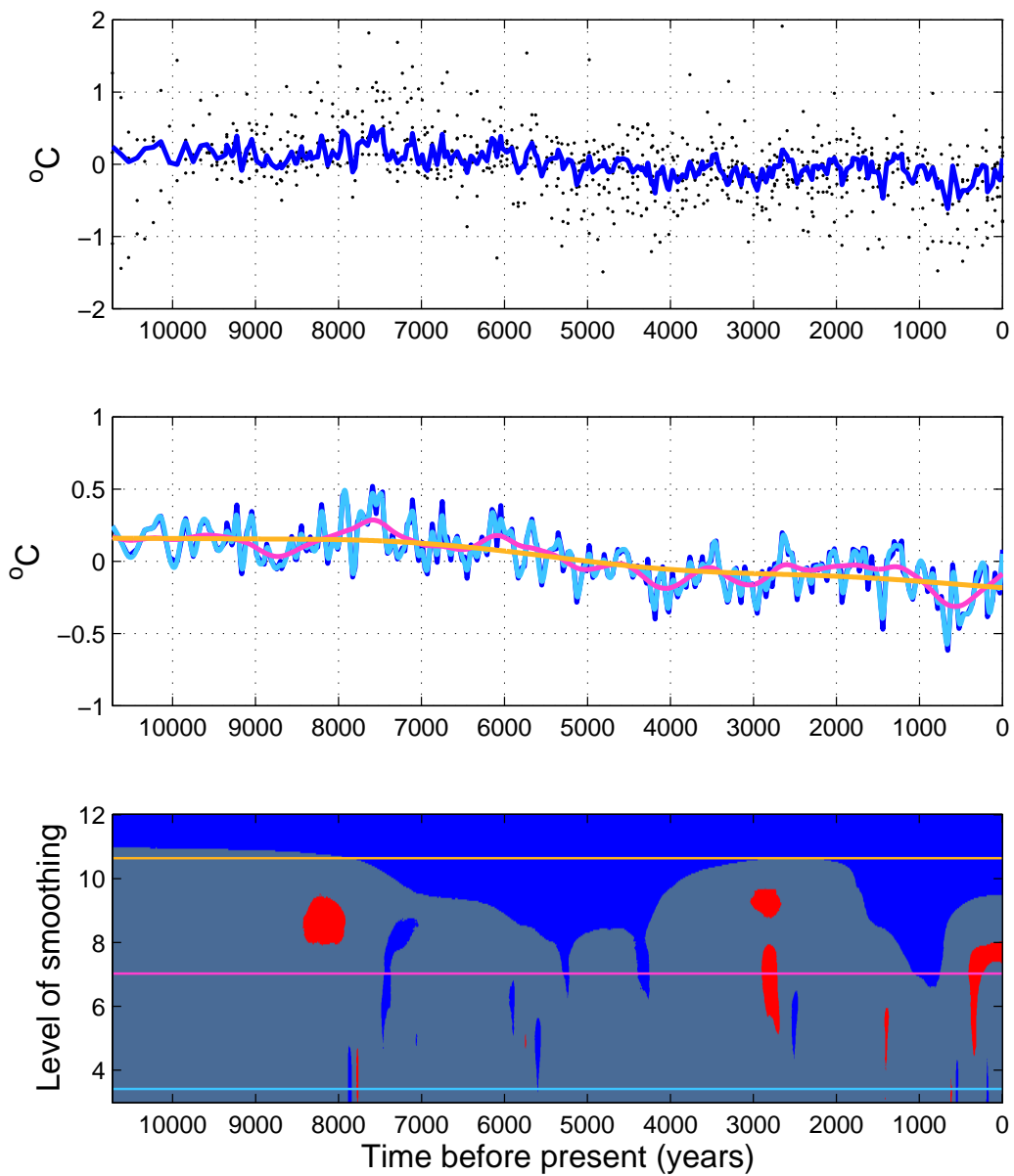

FiguRE S.7. Scale space analysis of the consensus of six temperature reconstructions. The model (6) in the main paper with large reconstruction errors is used and the level of credibility is $\alpha=0.8$. The top panel shows the reconstructions (dots) and the posterior mean of the consensus (blue curve). The middle panel shows the posterior mean of the consensus together with three smooths of the posterior consensus corresponding roughly to multi-decadal (light blue), centennial (purple), and millennial (yellow) time scales. The bottom panel is the credibility map where blue and red indicate credible cooling and warming, respectively. For more information see the text in the main paper. 

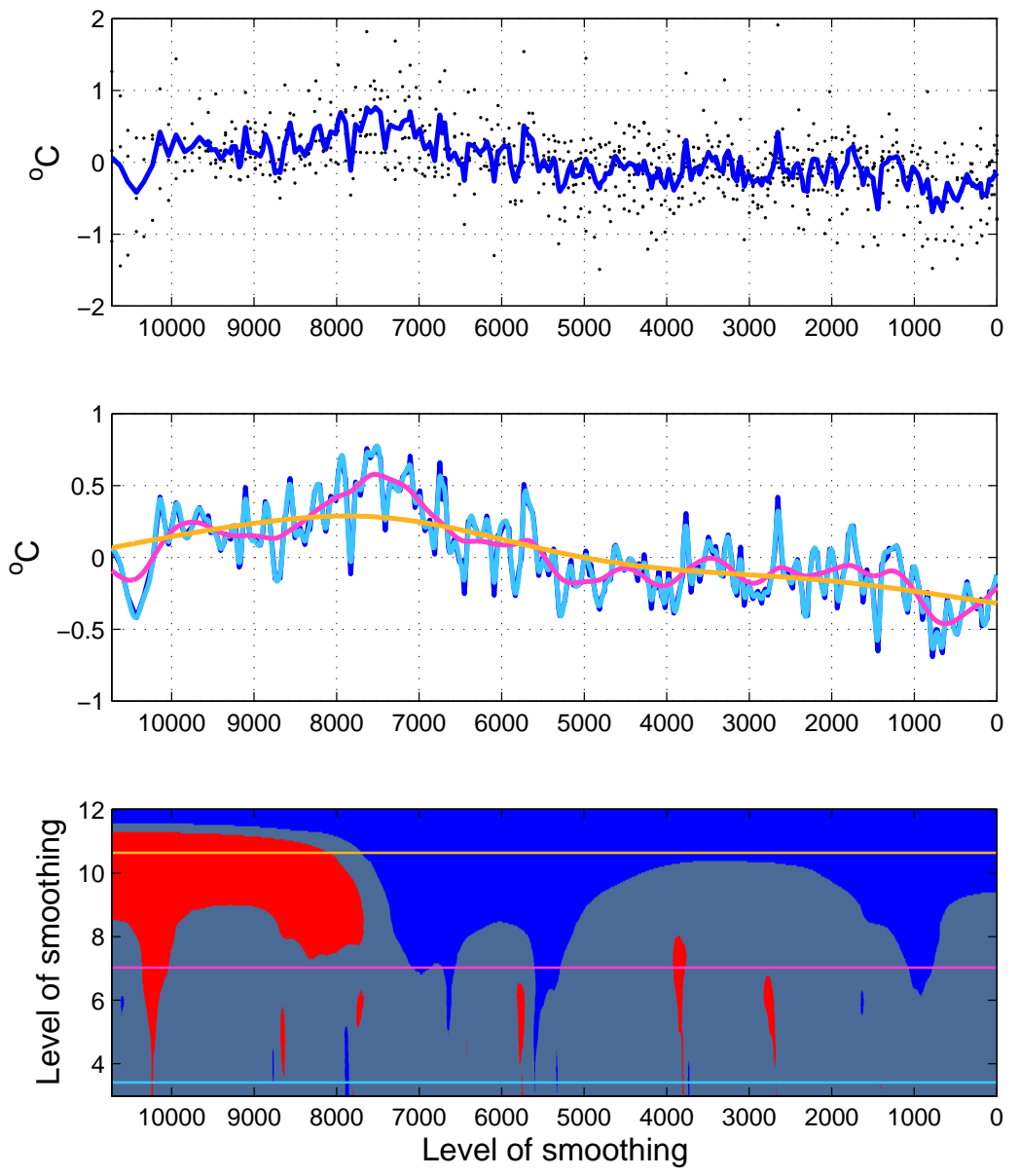

FiguRE S.8. Scale space analysis of the consensus of six temperature reconstructions. The model (6) in the main paper with small reconstruction errors is used and the level of credibility is $\alpha=0.8$. For more information see the caption of Figure S.7 and the text in the main paper. 
Table S.2. Sediment depths, calibrated ages, their standard errors and pollen-, chironomid- and diatom-based July mean temperature reconstructions for lakes Tsuolbmajavri and Toskal.

\begin{tabular}{|c|c|c|c|c|c|c|c|c|c|c|c|}
\hline & \multicolumn{5}{|c|}{ Lake Tsuolbmajavri } & & \multicolumn{5}{|c|}{ Lake Toskal } \\
\hline $\begin{array}{l}\text { depth } \\
\text { (cm) }\end{array}$ & $\begin{array}{c}\text { age } \\
\text { (cal BP) }\end{array}$ & $\begin{array}{c}\text { error } \\
\text { std }\end{array}$ & pollen & chironomids & diatoms & $\begin{array}{l}\text { depth } \\
(\mathrm{cm})\end{array}$ & $\begin{array}{c}\text { age } \\
\text { (cal BP) }\end{array}$ & $\begin{array}{c}\text { error } \\
\text { std }\end{array}$ & pollen & chironomids & diatoms \\
\hline 1 & 0 & 0 & 11.4 & 11.1 & 11.0 & 1 & 0 & 0 & 10.9 & 9.7 & 10.4 \\
\hline 3 & 92 & 17 & 11.6 & 11.0 & 10.9 & 2 & 30 & 38 & 11.2 & 10.1 & 9.5 \\
\hline 5 & 201 & 28 & 11.4 & 11.5 & 11.0 & 3 & 60 & 42 & 11.2 & 9.4 & 10.0 \\
\hline 7 & 310 & 38 & 11.3 & 10.9 & 10.9 & 4 & 120 & 47 & 10.7 & 9.4 & 9.9 \\
\hline 9 & 417 & 49 & 11.1 & 11.3 & 10.7 & 5 & 180 & 52 & 11.4 & 9.4 & 9.5 \\
\hline 11 & 524 & 60 & 11.1 & 11.1 & 10.5 & 6 & 240 & 56 & 11.0 & 9.6 & 10.2 \\
\hline 13 & 630 & 71 & 11.1 & 11.3 & 10.7 & 7 & 301 & 61 & 11.6 & 9.5 & 9.8 \\
\hline 15 & 734 & 82 & 11.1 & 11.1 & 10.7 & 8 & 361 & 65 & 11.2 & 9.6 & 9.8 \\
\hline 17 & 836 & 93 & 11.1 & 12.0 & 11.0 & 9 & 421 & 70 & 11.4 & 9.2 & 9.8 \\
\hline 19 & 936 & 104 & 11.4 & 11.2 & 10.7 & 10 & 482 & 75 & 10.5 & 9.6 & 9.8 \\
\hline 21 & 1034 & 114 & 12.0 & 11.1 & 10.8 & 11 & 542 & 79 & 10.6 & 9.9 & \\
\hline 23 & 1129 & 121 & 11.2 & 11.0 & 10.8 & 12 & 602 & 84 & 10.9 & & 10.1 \\
\hline 25 & 1222 & 127 & 11.9 & 11.5 & 11.2 & 13 & 662 & 88 & 10.9 & 9.3 & \\
\hline 27 & 1312 & 133 & 11.6 & 11.7 & 11.0 & 14 & 723 & 93 & 10.8 & & \\
\hline 29 & 1400 & 140 & 12.2 & 11.4 & 11.0 & 15 & 783 & 98 & 10.7 & & 9.8 \\
\hline 31 & 1486 & 146 & 11.2 & 11.0 & 11.0 & 16 & 843 & 102 & 11.0 & 9.8 & \\
\hline 33 & 1569 & 153 & 12.4 & 11.2 & 11.2 & 17 & 904 & 107 & 10.9 & & 10.0 \\
\hline 35 & 1650 & 159 & 12.0 & 11.1 & 11.0 & 18 & 964 & 111 & 10.8 & 10.0 & \\
\hline 37 & 1729 & 166 & 12.6 & 10.9 & 10.9 & 19 & 1024 & 116 & 11.8 & & \\
\hline 39 & 1805 & 172 & 12.7 & 11.4 & 11.0 & 20 & 1084 & 121 & 10.9 & & 9.8 \\
\hline 41 & 1879 & 177 & 11.5 & 11.3 & 11.0 & 21 & 1145 & 123 & 11.9 & 9.8 & \\
\hline 43 & 1952 & 180 & 12.5 & 10.8 & 10.9 & 22 & 1205 & 123 & 11.5 & 9.9 & 9.7 \\
\hline 45 & 2022 & 183 & 11.4 & 12.0 & 10.9 & 23 & 1265 & 122 & 11.4 & 9.8 & \\
\hline 47 & 2090 & 185 & 11.2 & 10.7 & 11.2 & 24 & 1325 & 122 & 11.7 & 9.8 & \\
\hline 49 & 2157 & 188 & 12.0 & 10.6 & 10.9 & 25 & 1386 & 122 & 11.4 & 9.4 & 10.1 \\
\hline 51 & 2221 & 190 & 12.7 & 10.6 & 11.0 & 26 & 1446 & 121 & 10.6 & 9.4 & \\
\hline 53 & 2285 & 193 & 12.6 & 10.3 & 11.2 & 27 & 1506 & 121 & 11.2 & 9.8 & 9.9 \\
\hline 55 & 2346 & 195 & 11.8 & 10.1 & 11.0 & 28 & 1567 & 121 & 11.0 & 9.5 & \\
\hline 57 & 2407 & 198 & 12.9 & 10.1 & 10.9 & 29 & 1627 & 121 & 11.1 & 9.8 & \\
\hline 59 & 2466 & 200 & 12.4 & 10.8 & 11.0 & 30 & 1687 & 120 & 11.3 & 10.0 & 10.1 \\
\hline 61 & 2523 & 202 & 12.0 & 10.8 & 10.9 & 31 & 1747 & 120 & 11.7 & 10.1 & \\
\hline 63 & 2580 & 199 & 12.0 & 10.7 & 11.0 & 32 & 1808 & 120 & 11.2 & 9.9 & \\
\hline 65 & 2636 & 196 & 12.3 & 10.9 & 11.1 & 33 & 1868 & 119 & 11.1 & 9.5 & 9.8 \\
\hline 67 & 2690 & 194 & 12.6 & 10.4 & 10.9 & 34 & 1928 & 119 & 11.2 & 9.4 & \\
\hline 69 & 2744 & 191 & 11.9 & 10.7 & 11.1 & 35 & 1989 & 119 & 11.9 & 9.6 & 9.9 \\
\hline 71 & 2798 & 189 & 12.6 & 10.2 & 11.1 & 36 & 2049 & 119 & 11.5 & 9.9 & \\
\hline 73 & 2850 & 186 & 11.9 & 10.3 & 10.7 & 37 & 2109 & 118 & 11.1 & 9.8 & 9.8 \\
\hline 75 & 2902 & 183 & 12.0 & 10.4 & 11.0 & 38 & 2169 & 118 & 11.2 & 9.9 & \\
\hline 77 & 2953 & 181 & 11.9 & 10.5 & 10.6 & 39 & 2230 & 118 & 11.7 & 9.7 & \\
\hline 79 & 3004 & 178 & 12.0 & 10.3 & 10.7 & 40 & 2290 & 117 & 10.7 & 9.4 & 9.7 \\
\hline 81 & 3055 & 178 & 11.1 & 10.9 & 10.7 & 41 & 2350 & 117 & 11.4 & 9.8 & \\
\hline 83 & 3106 & 185 & 12.8 & 10.9 & 11.1 & 42 & 2411 & 117 & 11.8 & 9.6 & 9.8 \\
\hline 85 & 3156 & 192 & 12.0 & 10.5 & 10.6 & 43 & 2471 & 119 & 11.9 & 9.5 & \\
\hline 87 & 3206 & 198 & 12.2 & 11.3 & 10.8 & 44 & 2531 & 123 & 11.3 & 10.1 & \\
\hline 89 & 3256 & 205 & 12.6 & 11.4 & 10.9 & 45 & 2591 & 128 & 11.4 & 9.6 & 10.4 \\
\hline 91 & 3306 & 212 & 13.4 & 11.0 & 10.4 & 46 & 2652 & 132 & 13.2 & 9.9 & \\
\hline 93 & 3356 & 219 & 12.5 & 10.9 & 10.7 & 47 & 2712 & 136 & 11.7 & 10.0 & 9.8 \\
\hline 95 & 3406 & 225 & 12.8 & 11.0 & 10.8 & 48 & 2772 & 141 & 10.9 & 9.4 & \\
\hline 97 & 3456 & 232 & 12.2 & 10.8 & 11.0 & 49 & 2833 & 145 & 11.7 & & \\
\hline 99 & 3506 & 239 & 12.6 & 10.8 & 10.8 & 50 & 2893 & 149 & 11.1 & & 9.8 \\
\hline 101 & 3556 & 245 & 12.9 & 10.5 & 10.9 & 51 & 2953 & 153 & 11.3 & 9.7 & \\
\hline 103 & 3606 & 251 & 12.2 & 10.5 & 10.8 & 52 & 3013 & 158 & 11.5 & & 9.9 \\
\hline 105 & 3657 & 256 & 12.1 & 10.6 & 10.9 & 53 & 3074 & 162 & 11.3 & 9.8 & \\
\hline 107 & 3708 & 262 & 12.0 & 10.4 & 10.7 & 54 & 3134 & 166 & 11.6 & & \\
\hline 109 & 3759 & 267 & 13.4 & 11.0 & 10.8 & 55 & 3194 & 171 & 11.0 & & 10.0 \\
\hline 111 & 3810 & 273 & 11.9 & 10.5 & 10.8 & 56 & 3255 & 175 & 11.6 & 9.9 & \\
\hline 113 & 3862 & 278 & 11.6 & 10.5 & 10.7 & 57 & 3315 & 179 & 11.4 & & 9.8 \\
\hline 115 & 3914 & 284 & 11.5 & 10.3 & 10.6 & 58 & 3375 & 184 & 11.5 & 9.5 & \\
\hline 117 & 3966 & 289 & 12.3 & 10.3 & 10.8 & 59 & 3435 & 188 & 11.7 & & \\
\hline 119 & 4019 & 295 & 11.7 & 10.0 & 10.7 & 60 & 3496 & 192 & 11.6 & & 10.1 \\
\hline 121 & 4072 & 299 & 12.0 & 10.4 & 10.9 & 61 & 3556 & 196 & 11.3 & 9.9 & \\
\hline 123 & 4125 & 297 & 12.1 & 9.9 & 10.8 & 62 & 3616 & 198 & 11.7 & & 10.2 \\
\hline 125 & 4179 & 295 & 12.2 & 10.0 & 10.5 & 63 & 3677 & 200 & 11.5 & 9.9 & \\
\hline 127 & 4233 & 294 & 12.4 & 9.8 & 10.6 & 64 & 3737 & 202 & 11.4 & & \\
\hline 129 & 4288 & 292 & 11.6 & 10.4 & 10.8 & 65 & 3797 & 204 & 11.9 & & 9.9 \\
\hline 131 & 4343 & 291 & 12.2 & 10.3 & 10.8 & 66 & 3857 & 206 & 11.5 & 10.2 & \\
\hline 133 & 4398 & 289 & 11.7 & 10.7 & 10.8 & 67 & 3918 & 208 & 11.6 & & 10.1 \\
\hline 135 & 4454 & 287 & 12.0 & 10.3 & 10.7 & 68 & 3978 & 211 & 11.7 & 10.0 & \\
\hline 137 & 4511 & 286 & 12.1 & 10.4 & 11.1 & 69 & 4038 & 213 & 11.9 & & \\
\hline
\end{tabular}




\begin{tabular}{|c|c|c|c|c|c|c|c|c|c|c|c|}
\hline 139 & 4568 & 284 & 12.1 & 10.5 & 11.0 & 70 & 4099 & 215 & 11.8 & & 10.0 \\
\hline 141 & 4625 & 285 & 12.7 & 10.7 & 10.8 & 71 & 4159 & 217 & 11.5 & 10.0 & \\
\hline 143 & 4683 & 294 & 12.1 & 10.2 & 11.0 & 72 & 4219 & 219 & 11.5 & 10.1 & 9.8 \\
\hline 145 & 4742 & 303 & 12.4 & 10.6 & 10.7 & 73 & 4279 & 221 & 12.0 & 10.4 & \\
\hline 147 & 4801 & 311 & 11.9 & 10.7 & 11.2 & 74 & 4340 & 224 & 12.0 & 10.0 & \\
\hline 149 & 4860 & 320 & 11.8 & 10.7 & 10.9 & 75 & 4400 & 226 & 12.1 & 10.4 & 9.8 \\
\hline 151 & 4920 & 329 & 12.3 & 11.3 & 10.9 & 76 & 4460 & 228 & 11.9 & 10.4 & \\
\hline 153 & 4981 & 337 & 13.6 & 10.0 & 11.0 & 77 & 4520 & 230 & 12.3 & 10.3 & 10.0 \\
\hline 155 & 5043 & 346 & 12.3 & 10.4 & 11.0 & 78 & 4581 & 232 & 12.1 & 10.1 & \\
\hline 157 & 5105 & 355 & 11.9 & 11.0 & 10.9 & 79 & 4641 & 234 & 11.1 & 10.0 & \\
\hline 159 & 5167 & 364 & 12.5 & 11.0 & 10.8 & 80 & 4701 & 236 & 10.9 & 10.1 & 9.9 \\
\hline 161 & 5230 & 370 & 11.4 & 10.4 & 11.2 & 81 & 4762 & 239 & 11.3 & 9.7 & \\
\hline 163 & 5294 & 367 & 11.4 & 10.6 & 10.9 & 82 & 4822 & 241 & 10.1 & 10.1 & 10.0 \\
\hline 165 & 5359 & 365 & 12.4 & 10.8 & 11.3 & 83 & 4882 & 243 & 9.8 & 10.0 & \\
\hline 167 & 5424 & 363 & 11.5 & 11.3 & 11.1 & 84 & 4942 & 245 & 11.1 & 9.6 & \\
\hline 169 & 5490 & 360 & 11.9 & 10.9 & 11.0 & 85 & 5003 & 247 & 11.0 & & 9.7 \\
\hline 171 & 5556 & 358 & 11.8 & 10.9 & 10.8 & 86 & 5063 & 249 & 10.5 & 9.9 & \\
\hline 173 & 5623 & 356 & 12.9 & 11.2 & 10.9 & 87 & 5123 & 251 & 11.0 & & 9.7 \\
\hline 175 & 5691 & 353 & 12.7 & 11.0 & 11.1 & 88 & 5184 & 253 & 11.3 & 10.0 & \\
\hline 177 & 5760 & 351 & 12.2 & 10.5 & 10.9 & 89 & 5244 & 254 & 11.3 & & \\
\hline 179 & 5829 & 349 & 11.6 & 10.5 & 10.9 & 90 & 5304 & 255 & 11.8 & & 10.3 \\
\hline 181 & 5899 & 347 & 12.2 & 10.5 & 11.0 & 91 & 5364 & 256 & 11.9 & 9.6 & \\
\hline 183 & 5970 & 346 & 12.3 & 11.3 & 11.1 & 92 & 5425 & 257 & 11.6 & & 10.0 \\
\hline 185 & 6042 & 344 & 12.2 & 11.8 & 11.4 & 93 & 5485 & 258 & 11.4 & 10.0 & \\
\hline 187 & 6114 & 343 & 12.2 & 11.2 & 11.5 & 94 & 5545 & 259 & 11.4 & & \\
\hline 189 & 6187 & 342 & 12.8 & 10.9 & 11.1 & 95 & 5606 & 260 & 12.0 & & 10.1 \\
\hline 191 & 6261 & 341 & 12.8 & 10.9 & 11.2 & 96 & 5666 & 261 & 12.3 & 10.2 & \\
\hline 193 & 6335 & 339 & 12.5 & 11.2 & 11.1 & 97 & 5726 & 262 & 13.3 & & 10.0 \\
\hline 195 & 6411 & 338 & 13.2 & 11.4 & 10.9 & 98 & 5786 & 263 & 11.9 & 9.7 & \\
\hline 197 & 6487 & 337 & 12.2 & 11.4 & 11.0 & 99 & 5847 & 264 & 11.9 & & \\
\hline 199 & 6564 & 336 & 12.9 & 10.5 & 11.1 & 100 & 5907 & 265 & 12.1 & & 10.2 \\
\hline 201 & 6641 & 336 & 12.3 & 10.8 & 10.8 & 101 & 5967 & 266 & 11.6 & 10.2 & \\
\hline 203 & 6720 & 342 & 12.7 & 10.8 & 10.8 & 102 & 6028 & 267 & 11.5 & & 10.1 \\
\hline 205 & 6799 & 349 & 12.9 & 10.9 & 11.3 & 103 & 6088 & 268 & 11.4 & 10.1 & \\
\hline 207 & 6879 & 355 & 12.1 & 11.3 & 11.1 & 104 & 6148 & 269 & 11.5 & & \\
\hline 209 & 6959 & 361 & 12.6 & 11.5 & 10.8 & 105 & 6208 & 270 & 11.7 & & 9.9 \\
\hline 211 & 7041 & 367 & 13.2 & 11.3 & 10.8 & 106 & 6269 & 271 & 12.1 & 10.0 & \\
\hline 213 & 7123 & 373 & 13.1 & 11.3 & 11.0 & 107 & 6329 & 272 & 12.2 & & 9.5 \\
\hline 215 & 7206 & 379 & 13.3 & 11.3 & 10.9 & 108 & 6389 & 273 & 11.9 & 9.9 & \\
\hline 217 & 7289 & 385 & 13.9 & 10.9 & 10.7 & 109 & 6450 & 274 & 11.6 & & \\
\hline 219 & 7373 & 391 & 12.8 & 11.9 & 10.8 & 110 & 6510 & 275 & 11.2 & & 10.0 \\
\hline 221 & 7458 & 398 & 13.2 & 11.5 & 11.0 & 111 & 6570 & 276 & 12.1 & 10.2 & \\
\hline 223 & 7543 & 404 & 13.3 & 11.9 & 11.1 & 112 & 6630 & 277 & 11.7 & & 10.2 \\
\hline 225 & 7629 & 410 & 14.0 & 11.4 & 10.9 & 113 & 6691 & 278 & 12.5 & 10.0 & \\
\hline 227 & 7716 & 416 & 12.6 & 11.6 & 11.0 & 114 & 6751 & 279 & 12.4 & & \\
\hline 229 & 7803 & 422 & 12.9 & 11.1 & 10.7 & 115 & 6811 & 281 & 11.6 & & 9.9 \\
\hline 231 & 7891 & 428 & 12.9 & 11.7 & 11.3 & 116 & 6872 & 282 & 11.9 & 10.4 & \\
\hline 233 & 7980 & 435 & 13.6 & 11.4 & 10.9 & 117 & 6932 & 283 & 11.7 & & 9.8 \\
\hline 235 & 8069 & 442 & 13.0 & 11.7 & 11.2 & 118 & 6992 & 284 & 12.6 & 9.9 & \\
\hline 237 & 8159 & 448 & 12.5 & 11.2 & 11.3 & 119 & 7052 & 285 & 11.5 & & \\
\hline 239 & 8249 & 455 & 12.5 & 12.1 & 10.9 & 120 & 7113 & 287 & 12.3 & & 10.5 \\
\hline 241 & 8339 & 461 & 13.2 & 11.1 & 10.9 & 121 & 7173 & 288 & 11.9 & 10.2 & \\
\hline 243 & 8431 & 468 & 12.6 & 11.2 & 10.9 & 122 & 7233 & 289 & 13.1 & & 10.0 \\
\hline 245 & 8522 & 475 & 12.9 & 11.4 & 10.9 & 123 & 7294 & 290 & 12.3 & 10.0 & \\
\hline 247 & 8614 & 481 & 12.5 & 11.5 & 10.9 & 124 & 7354 & 290 & 12.0 & & \\
\hline 249 & 8707 & 488 & 12.0 & 11.3 & 10.8 & 125 & 7414 & 290 & 11.4 & & 10.1 \\
\hline 251 & 8800 & 496 & 11.7 & 11.7 & 10.6 & 126 & 7474 & 291 & 12.3 & 10.5 & \\
\hline 253 & 8893 & 508 & 12.6 & 11.2 & 10.8 & 127 & 7535 & 291 & 12.1 & & 10.4 \\
\hline 255 & 8987 & 521 & 12.6 & 11.6 & 10.6 & 128 & 7595 & 292 & 12.4 & 10.3 & \\
\hline 257 & 9081 & 533 & 12.0 & 11.6 & 11.0 & 129 & 7655 & 292 & 11.9 & & \\
\hline 259 & 9176 & 546 & 12.0 & 11.2 & 10.8 & 130 & 7716 & 293 & 12.1 & & 10.3 \\
\hline 261 & 9270 & 558 & 12.5 & 11.6 & 10.9 & 131 & 7776 & 293 & 12.6 & 10.0 & \\
\hline 263 & 9365 & 571 & 12.2 & 11.3 & 11.0 & 132 & 7836 & 293 & 12.3 & & 9.9 \\
\hline 265 & 9461 & 583 & 12.2 & 11.9 & 10.9 & 133 & 7896 & 294 & 12.1 & 9.9 & \\
\hline 267 & 9557 & 596 & 12.4 & 11.7 & 11.0 & 134 & 7957 & 294 & 12.1 & & \\
\hline 269 & 9653 & 608 & 12.3 & 11.9 & 11.0 & 135 & 8017 & 295 & 12.1 & & 10.0 \\
\hline 271 & 9749 & 622 & 12.3 & 11.5 & 10.9 & 136 & 8077 & 295 & 11.9 & 10.2 & \\
\hline 273 & 9845 & 638 & 12.3 & 10.9 & 11.2 & 137 & 8137 & 295 & 11.9 & & 9.9 \\
\hline 275 & 9942 & 654 & 12.2 & 12.4 & 10.7 & 138 & 8198 & 296 & 12.4 & 10.2 & \\
\hline 277 & 10039 & 670 & 11.7 & 11.5 & 10.9 & 139 & 8258 & 296 & 12.2 & & \\
\hline 279 & 10136 & 686 & 11.8 & 12.0 & 11.1 & 140 & 8318 & 297 & 11.1 & & 10.0 \\
\hline 281 & 10233 & 702 & 11.4 & 11.4 & 11.0 & 141 & 8379 & 297 & 11.4 & 10.0 & \\
\hline 283 & 10330 & 718 & 11.2 & 11.0 & 11.3 & 142 & 8439 & 297 & 11.4 & & 10.3 \\
\hline 285 & 10427 & 734 & 11.2 & 10.5 & 11.0 & 143 & 8499 & 298 & 11.8 & 9.6 & \\
\hline
\end{tabular}




\begin{tabular}{|c|c|c|c|c|c|c|c|c|c|c|c|}
\hline 287 & 10525 & 751 & 10.9 & 11.4 & 11.0 & 144 & 8559 & 298 & 11.9 & & \\
\hline 289 & 10622 & 767 & 10.8 & 11.9 & 11.0 & 145 & 8620 & 307 & 12.6 & & 9.9 \\
\hline \multirow[t]{16}{*}{291} & 10719 & 779 & 11.1 & 12.3 & 11.0 & 146 & 8680 & 323 & 11.3 & 9.6 & \\
\hline & & & & & & 147 & 8740 & 339 & 10.9 & & 10.0 \\
\hline & & & & & & 148 & 8801 & 355 & 11.3 & 9.9 & \\
\hline & & & & & & 149 & 8861 & 371 & 11.7 & & \\
\hline & & & & & & 150 & 8921 & 388 & 11.3 & & 9.9 \\
\hline & & & & & & 151 & 8981 & 404 & 11.4 & 9.9 & \\
\hline & & & & & & 152 & 9042 & 420 & 11.7 & & 10.4 \\
\hline & & & & & & 153 & 9102 & 436 & 11.9 & 9.9 & \\
\hline & & & & & & 154 & 9162 & 452 & 11.4 & & \\
\hline & & & & & & 155 & 9223 & 469 & 11.8 & & 10.5 \\
\hline & & & & & & 156 & 9283 & 485 & 11.3 & 10.0 & \\
\hline & & & & & & 157 & 9343 & 501 & 11.9 & & 10.4 \\
\hline & & & & & & 158 & 9403 & 517 & 11.6 & 10.0 & \\
\hline & & & & & & 159 & 9464 & 533 & 11.4 & & \\
\hline & & & & & & 160 & 9524 & 550 & 11.3 & & 10.3 \\
\hline & & & & & & 161 & 9584 & 558 & 11.4 & 10.1 & \\
\hline
\end{tabular}

